\title{
Rapid Reduction of Campylobacter Species in the Gut Microbiome of Preschool Children after Oral Azithromycin: A Randomized Controlled Trial
}

\author{
Armin Hinterwirth, ${ }^{1}$ Ali Sié, ${ }^{2}$ Boubacar Coulibaly, ${ }^{2}$ Lucienne Ouermi, ${ }^{2}$ Clarisse Dah, ${ }^{2}$ Charlemagne Tapsoba, ${ }^{2}$ Lina Zhong, ${ }^{1}$ \\ Cindi Chen, ${ }^{1}$ Thomas M. Lietman, ${ }^{1,3,4}$ Jeremy D. Keenan, ${ }^{1,3}$ Thuy Doan, ${ }^{1,3 *}$ and Catherine E. Oldenburg ${ }^{1,3,4}$ \\ ${ }^{1}$ Francis I. Proctor Foundation, University of California, San Francisco, San Francisco, California; ${ }^{2}$ Centre de Recherche en Santé de Nouna, \\ Nouna, Burkina Faso; ${ }^{3}$ Department of Ophthalmology, University of California, San Francisco, San Francisco, California; ${ }^{4}$ Department of \\ Epidemiology \& Biostatistics, University of California, San Francisco, San Francisco, California
}

\begin{abstract}
Campylobacter has emerged as a potential important cause of childhood morbidity in sub-Saharan Africa. Biannual mass azithromycin distribution has previously been shown to reduce all-cause child mortality in sub-Saharan Africa. We conducted a randomized controlled trial in Burkina Faso in which children were randomized in a 1:1 fashion to a 5 -day course of azithromycin or placebo to investigate the effect of oral antibiotics on the gut microbiome. We evaluated the changes in the gut microbiome of preschool children treated with azithromycin using metagenomic DNA sequencing. We found that three Campylobacter species were reduced with azithromycin treatment compared with placebo. These results were consistent with other studies that have shown decreases in Campylobacter species after azithromycin treatment, generating the hypothesis that a decrease in Campylobacter may contribute to observations of reduction in mortality following azithromycin distribution.
\end{abstract}

\section{INTRODUCTION}

Mass azithromycin distribution has been shown to reduce all-cause child mortality in some settings in sub-Saharan Africa. ${ }^{1,2}$ The precise mechanism behind this action is unclear but presumably is mediated through the effect of azithromycin on pathogenic organisms, including those that cause diarrhea. Although there has been a reduction in diarrhea-related mortality in children younger than 5 years, it remains an important cause of death in children in sub-Saharan Africa. ${ }^{3}$ Recently, Campylobacter species have been shown to be of increasing importance in the etiology of childhood diarrhea infection in developing settings. ${ }^{4,5}$ Given concerns related to fluoroquinolone resistance, azithromycin is the first-line treatment for campylobacteriosis requiring antibiotic treatment. ${ }^{6} \mathrm{~A}$ reduction in Campylobacter species was recently demonstrated in communities receiving mass azithromycin distribution compared with placebo in Niger. ${ }^{7,8}$ Hypothetically, a population reduction in Campylobacter species burden may reduce transmission in communities, which may reduce diarrhea-related mortality.

Several strategies for the distribution of azithromycin for the prevention of child mortality are being considered. Mass treatment has advantages in that entire communities can be treated efficiently at a single time point. Alternative strategies could include distribution from a fixed point, such as integration with childhood vaccination visits. ${ }^{9}$ Individual distribution may affect transmission or burden of pathogens differently than community distribution. Here, we evaluated the short-term effect of a course of azithromycin compared with placebo on the detection of Campylobacter species in preschool children in rural Burkina Faso individually randomized to azithromycin or placebo.

\section{METHODS}

Participants. This is a secondary analysis of a randomized controlled trial designed to evaluate the effect of a single

*Address correspondence to Thuy Doan, Francis I. Proctor Foundation, University of California, San Francisco, 513 Parnassus Ave., Rm. S309, San Francisco, CA 94143. E-mail: thuy.doan@ucsf.edu course of antibiotics on the gut microbiome in children aged 6-59 months in Nouna district, Burkina Faso, in July $2017 .{ }^{10}$ The study took place in two communities in the Nouna Health and Demographic Surveillance System in rural northwestern Burkina Faso. Households with at least two children younger than 5 years based on the most recent Health and Demographic Surveillance Site census were eligible for inclusion. Children were eligible if they were aged between 6 and 59 months and with parental consent. There were no exclusions based on preexisting morbidity or antibiotic use. The study was approved by the institutional review boards at the University of California San Francisco, San Francisco, CA, and the Centre de Recherche en Santé de Nouna in Nouna, Burkina Faso. Written informed consent was obtained from the caregiver of each child.

Trial design. Complete trial methods have been previously reported. ${ }^{10-12}$ Households were randomized in a 1:1:1:1 fashion to a 5-day course of azithromycin, amoxicillin, cotrimoxazole, or placebo. In households with more than two children, two children were randomly chosen to participate in the study from each household. In each household, one child was randomly assigned to receive antibiotic and the other to placebo (in placebo households, both children received placebo). The present analysis was limited only to households randomized to azithromycin or placebo, as the study question was related to the effect of azithromycin on Campylobacter species, and only to children who received the household's random treatment assignment (e.g., in azithromycin households, the child who received azithromycin, and in placebo households, one child who had received placebo). We focused on the azithromycin arm because azithromycin distribution was associated with a reduction of Campylobacter in the gut of children in Niger ${ }^{7,8}$ although it was unclear if the same effect would be seen in Burkina Faso. Children were evaluated at baseline (pretreatment) and 5 days after the last antibiotic dose (posttreatment).

Intervention. Study medication was prepared as pediatric oral suspension, and dosing was weight based using baseline anthropometric assessments. Azithromycin dosing was based on the lower end of the standard pediatric dosing for mild-to-moderate infection (one $10 \mathrm{mg} / \mathrm{kg}$ dose on the first day followed by $5 \mathrm{mg} / \mathrm{kg}$ daily for 4 days). Study staff prepared 
placebo as a mixture of powdered milk, sugar, and bottled water. Study medications were prepared in opaque syringes labeled for each child, prepared fresh each day. Treatment was administered from a central point in each community, with a community mobilizer visiting the homes of participating children to ask them to come to the distribution point for treatment. All study doses were directly observed, and treatment was recorded for each child, including reasons for missing a treatment.

Outcome assessment. Rectal samples were collected in the field during the baseline and posttreatment visits and placed on Stool Nucleic Acid Collection and Transport Tube with Norgen Stool Preservative (Norgen, Ontario, Canada) at ambient temperature. Samples were stored at the Centre de Recherche en Santé de Nouna laboratory at the end of each collection day at $-80^{\circ} \mathrm{C}$ until they were shipped to the United States. Samples were shipped on ice packs and stored at $-80^{\circ} \mathrm{C}$ at the Proctor Foundation until processing. Samples were placed in a random order before processing and were deidentified, and all laboratory personnel were masked to the child's treatment assignment. Thirty samples from each arm were subjected to DNA-seq. ${ }^{12}$ Briefly, DNA was extracted using the Norgen stool DNA isolation kits (Norgen) as per the manufacturer's instructions. Each sample was quantified using the Qubit ${ }^{\circledR}$ RNA HS Assay Kit (ThermoFisher Scientific, Waltham, $\mathrm{MA})$, and $25 \mathrm{ng}$ of DNA was used to prepare sequencing library after fragmentation using the NEBNext Ultra II DNA Library Prep Kit (E7645, New England BioLabs, Ipswich, MA) according to the manufacturer's recommendation and then amplified with 11 PCR cycles. Samples were sequenced on the NovaSeq using 150-nucleotide paired-end sequencing.

Data analysis. Sequencing reads were analyzed using an in-house computational pipeline. ${ }^{7}$ Briefly, an initial humansequence removal step was accomplished as previously described. ${ }^{13}$ Unaligned reads were quality filtered using PriceSeqFilter with the "-rnf 90" and "-rqf 850.98 " settings. ${ }^{14}$ Reads passing quality control were then subjected to duplicate removal. Paired reads were then assessed for complexity by compression with the Lempel-Ziv-Welch algorithm. ${ }^{15}$
Another round of human reads removal was performed using the very sensitive local mode of Bowtie2 (v. 2.2.4) with the same hg38 and Pantroglodytes genome (panTro4, 2011, UCSC). ${ }^{16}$ The remaining nonhost read pairs were then passed onto Centrifuge (1.0.3) to align to the entire NCBI nonredundant reference database. DESeq2 (v. 1.22.1) was used to compare the relative changes in abundances of individual microbial taxa between the treatment groups at the genus and species levels. ${ }^{17}$ Only taxa that presented in $25 \%$ or greater in the dataset were used. Differentially abundant taxa with a false discovery rate $<0.01$ and a 4 -fold change were considered as notable.

\section{RESULTS}

Of 62 enrolled children who were randomized to either azithromycin or placebo, 31 children in the azithromycin arm and 30 children in the placebo arm had a rectal swab collected at both baseline and posttreatment (Figure 1). Baseline characteristics for the study population have been previously reported. ${ }^{12}$ At the genus level, a single 5-day course of azithromycin resulted in a reduction of 10 genera compared with placebo, including Campylobacter (Figure 2A). At a higher resolution at the species level, 48 organisms were differentially detected in the gut between treatment arms (Figure 2B and C). Of those organisms, Campylobacter jejuni, Campylobacter ureolyticus, and Campylobacter hominis were reduced in children treated with azithromycin (Figure 2B). At baseline, these same Campylobacter species were not statistically different between groups.

\section{DISCUSSION}

Mass drug distribution of azithromycin reduces childhood mortality in Nigerien preschool children in a large simple randomized controlled trial. Although the effects of azithromycin on childhood mortality is likely to be multifactorial, a reduction of abundance in Campylobacter species was seen at 6 months after the fourth biannual mass azithromycin distribution at the

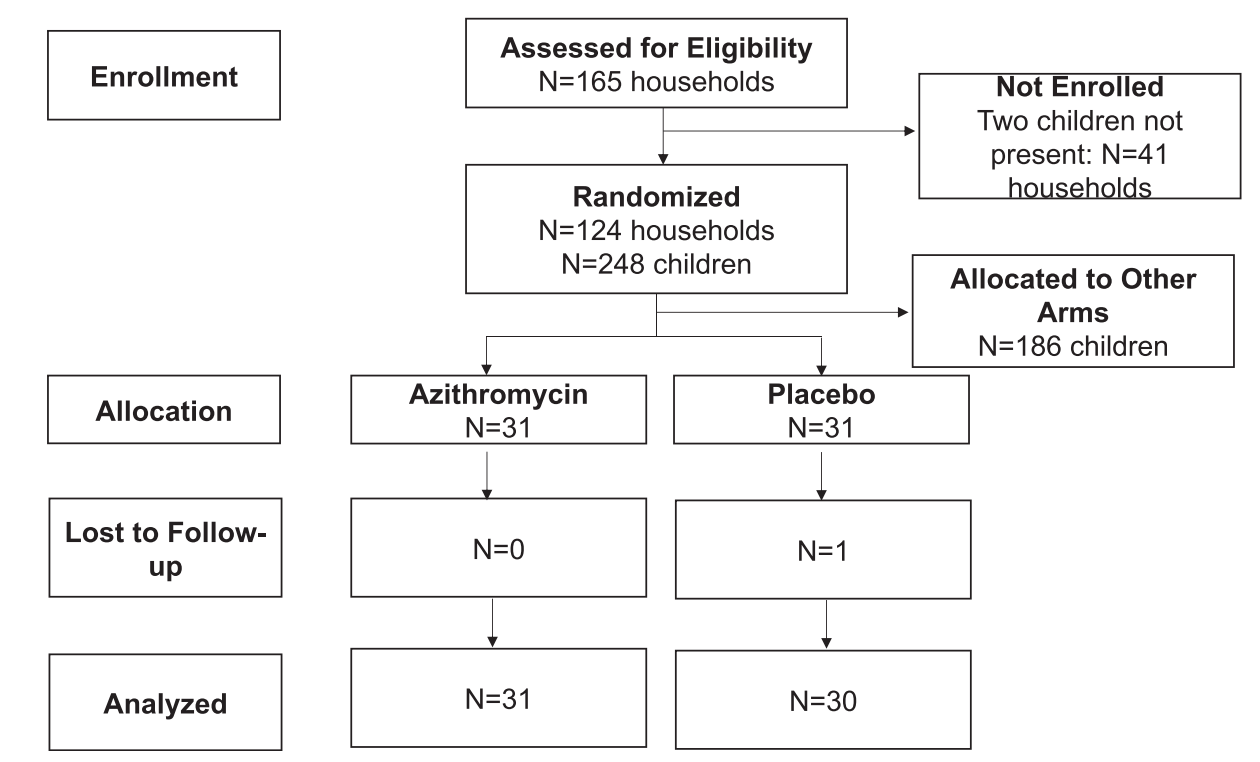

FIGURE 1. Consolidated Standards Of Reporting Trials (CONSORT) flow diagram of enrolled participants. 
A

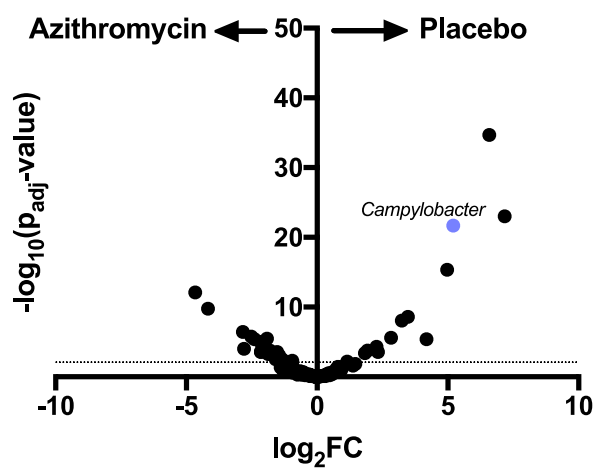

B

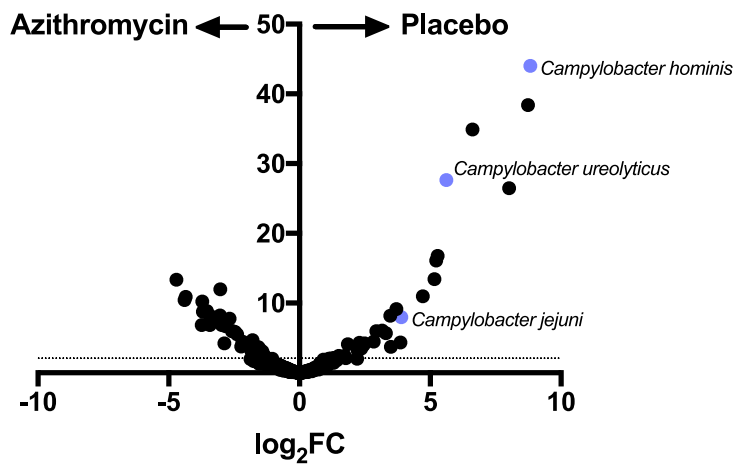

C

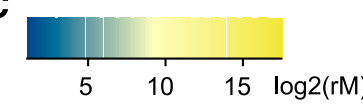

Placebo Azithromycin
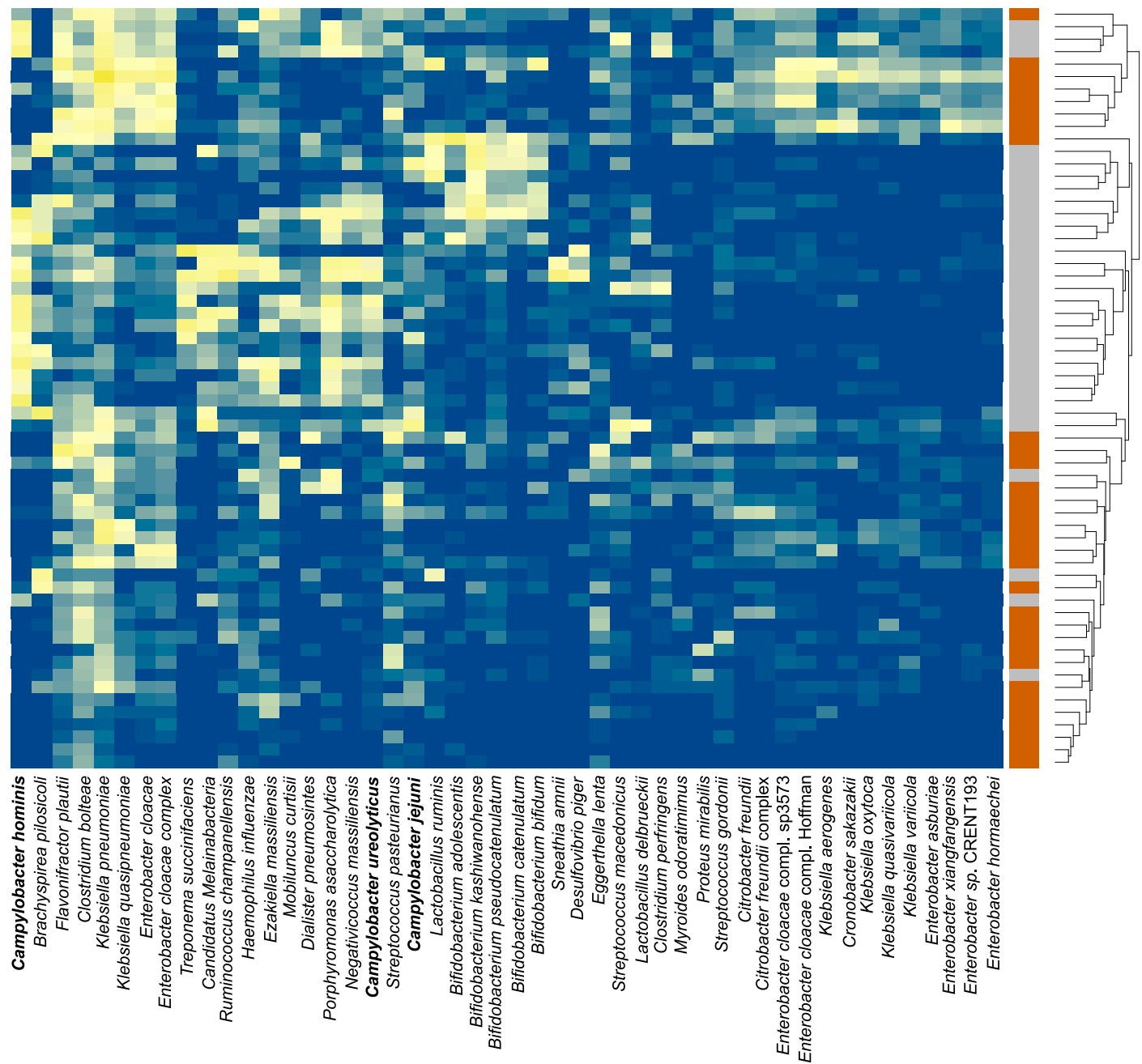

FIGURE 2. Effects of azithromycin on the gut microbiome of preschool children. Volcano plot of relative abundance distribution of gut bacteria at the genus level (A) and at the species level (B). (C) Normalized abundance, arranged by unsupervised hierarchical clustering, reflecting increased abundance (yellow) or decreased abundance (blue) of species (columns) for each child (rows). Forty-eight differential species identified with a false discovery rate $<0.01$ and log ${ }_{2} \mathrm{FC}>2$ (two-sided negative binomial Wald test with Benjamini-Hochberg correction for multiple comparisons). Campylobacter species are bolded. This figure appears in color at www.ajtmh.org.

community level. In a separate study in the same region of Niger, individually treated children also showed a relative reduction in Campylobacter at the genus level 5 days after azithromycin treatment compared with the placebo-treated children. ${ }^{8}$ Therefore, Campylobacter as a group appeared to be strongly modulated by azithromycin shortly after treatment, and this effect is also prolonged to 6 months after the last treatment in preschool children in Niger. 
Here, we showed that the abundance of three Campylobacter species (C. jejuni, C. ureolyticus, and C. hominis) was acutely reduced in individually azithromycin-treated children in Burkina Faso. Whereas Campylobacter jejuni is well documented to cause diarrhea and neurological sequelae, the role of Campylobacter hominis and Campylobacter ureolyticus in gastrointestinal disturbances has not been appreciated until recently. In a study by Bullman and others, ${ }^{18}$ Campylobacter ureolyticus-related campylobacteriosis was noted to be the second highest causative agent, higher than that of Campylobacter coli, in Southern Ireland. In addition, Campylobacter hominis and Campylobacter ureolyticus have been associated with inflammatory states such as ulcerative colitis. ${ }^{19,20}$ We were, however, unable to detect a difference in diarrheal episodes between treatment groups. ${ }^{11}$ Campylobacter hominis and Campylobacter ureolyticus had higher fold changes and typically are not associated with acute diarrhea, which may partially explain this finding.

Burkina Faso shares a border with Niger, and the two countries are similar geographically. In this study area, approximately one-third of the population practices open defecation. ${ }^{21}$ Therefore, Campylobacter transmission through human-to-human interactions may be more prevalent than what is observed in the developed world, where most of the transmission is through contaminated livestock such as chicken. In the rural setting of Burkina Faso, treatment at the individual or community level may equally have the potential to decrease transmission and improve childhood mortality, although at the cost of increasing macrolide resistance. ${ }^{12}$

Limitations of this study include the short duration of followup of 5 days and the sample storage condition that prevented phenotypic analysis of the gut microorganisms. In addition, the small number of children, coupled with the short study duration, precludes mortality analysis. Longer term studies are needed to evaluate the repopulation of Campylobacter species after antibiotic intake. Although we asked caregivers about adverse events including diarrhea during treatment, ${ }^{11}$ this study was not designed to measure clinical diarrhea, and, thus, this study was unable to detect any effect of treatment on Campylobacter-related diarrhea.

In summary, a single course of azithromycin decreased the load of multiple Campylobacter species in the gut microbiome of preschool children in Burkina Faso. It remains to be seen if this acute change translates to a biologically relevant effect such as a reduction in childhood mortality or improved health outcomes for children.

Received December 17, 2019. Accepted for publication May 4, 2020.

Published online June 8, 2020.

Financial support: T. D. and C. E. O. were supported in part by a Research to Prevent Blindness Career Development Award.

Authors' addresses: Armin Hinterwirth, Lina Zhong, Cindi Chen, Thuy Doan, Thomas M. Lietmanm, Jeremy D. Keenan, and Catherine E. Oldenburg, Francis I. Proctor Foundation, University of California, San Francisco, San Francisco, CA, E-mails: armin.hinterwirth@ucsf.edu, lina.zhong@ucsf.edu, cindi.chen@ucsf.edu, thuy.doan@ucsf.edu, tom.lietman@ucsf.edu, jeremy.keenan@ucsf.edu, and catherine. oldenburg@ucsf.edu. Ali Sié, Boubacar Coulibaly, Lucienne Ouermi, Clarisse Dah, and Charlemagne Tapsoba, Centre de Recherche en
Santé de Nouna, Nouna, Burkina Faso, E-mails: sieali@yahoo.fr, boubacar@fasonet.bf, ouermil@yahoo.fr, n.clarissedah@yahoo.fr, and charlemagnetapsoba@gmail.com.

\section{REFERENCES}

1. Keenan JD et al., 2018. Azithromycin to reduce childhood mortality in sub-Saharan Africa. N Engl J Med 378: 1583-1592.

2. Keenan JD et al., 2019. Longer-term assessment of azithromycin for reducing childhood mortality in Africa. N Engl J Med 380: 2207-2214.

3. Liu L, Oza S, Hogan D, Chu Y, Perin J, Zhu J, Lawn JE, Cousens S, Mathers C, Black RE, 2016. Global, regional, and national causes of under- 5 mortality in 2000-15: an updated systematic analysis with implications for the sustainable development goals. Lancet 388: 3027-3035.

4. Kaakoush NO, Castaño-Rodríguez N, Mitchell HM, Man SM, 2015. Global epidemiology of Campylobacter infection. Clin Microbiol Rev 28: 687-720.

5. Francois $\mathrm{R}$ et al., 2018. The other Campylobacters : not innocent bystanders in endemic diarrhea and dysentery in children in low-income settings. PLoS Negl Trop Dis 12: e0006200.

6. Nachamkin I, Ung H, Li M, Study T, 2002. Fluoroquinolone resistance in Campylobacter. Emerg Infect Dis 8: 1501-1503.

7. Doan T et al., 2019 Gut microbiome alteration in MORDOR I: a community-randomized trial of mass azithromycin distribution. Nat Med 25: 1370-1376.

8. Doan T et al., 2017. Gut microbial diversity in antibiotic-naïve children after systemic antibiotic exposure: a randomized controlled trial. Clin Infect Dis 64: 1147-1153.

9. Sié A et al., 2019. A double-masked placebo-controlled trial of azithromycin to prevent child mortality in Burkina Faso, west Africa: community health with azithromycin trial (CHAT) study protocol. Trials 20: 675 .

10. Oldenburg CE et al., 2018. Effect of commonly-used pediatric antibiotics on gut microbial diversity in preschool children in Burkina Faso: a randomized clinical trial. Open Forum Infect Dis 5: ofy289.

11. Sie A et al., 2018. Effect of antibiotics on short-term growth among children in Burkina Faso: a randomized trial. Am J Trop Med Hyg 99: 789-796.

12. Oldenburg CE et al., 2020. Gut resistome after oral antibiotics in preschool children in Burkina Faso: a randomized controlled trial. Clin Infect Dis 70: 525-527.

13. Doan T et al., 2018. Mass azithromycin distribution and community microbiome: a cluster-randomized trial. Open Forum Infect Dis 5: ofy 182.

14. Doan $T$ et al., 2017. Metagenomic DNA sequencing for the diagnosis of intraocular infections. Ophthalmology 124: 1247-1248.

15. Ziv J, Lempel A, 1977. A universal algorithm for sequential data compression. IEEE Trans Inf Theory 23: 337-343.

16. Langmead B, Salzberg SL, 2013. Fast gapped-read alignment with Bowtie 2. Nat Methods 9: 357-359.

17. Love MI, Huber W, Anders S, 2014. Moderated estimation of fold change and dispersion for RNA-seq data with DESeq2. Genome Biol 15: 550.

18. Bullman S, Corcoran D, O'Leary J, O'Hare D, Lucey B, Sleator RD, 2011. Emerging dynamics of human campylobacteriosis in southern Ireland. FEMS Immunol Med Microbiol 63: 248-253.

19. O'Donovan D, Corcoran GD, Lucey B, Sleator RD, 2014. Campylobacter ureolyticus: a portrait of the pathogen. Virulence 5: 498-506.

20. Mukhopadhya I, Thomson JM, Hansen R, Berry SH, El-omar EM, Hold GL, 2011. Detection of Campylobacter concisus and other Campylobacter species in colonic biopsies from adults with ulcerative colitis. PLoS One 6: e21490.

21. Dennis EG et al., 2019. Short-term weight gain among preschool children in rural Burkina Faso: a secondary analysis of a randomised controlled trial. BMJ Open 9: e029634. 